\title{
BIOAUGMENTATION APPROACH IN RHIZOSPHERIC MICROBIOME RESEARCH: A LESSON FROM ARSENIC REMEDIATION
}

\author{
Md. Mahmudul Hasan*
}

Department of Nutrition and Food Technology, Jessore University of Science and Technology, Jessore-7408, Bangladesh. Editor-in-Chief, Malaysian Journal of Halal Research

*To whom correspondence should be addressed : drhasan_nft@just.edu.bd; hasanm_agb@yahoo.com

This is an open access article distributed under the Creative Commons Attribution License, which permits unrestricted use, distribution, and reproduction in any medium, provided the original work is properly cited.

\section{ARTICLE DETAILS}

Edited by:

Muhammad Azhar Ali

University of Agriculture Faisalabad

38080 Punjab, Pakistan.

Email: azhar_ali@uaf.edu.pk

Reviewed by :

Mudassir Arif

University of Agriculture Faisalabad 38080 Punjab, Pakistan

Email: mudassir.arif@uaf.edu.pk

\section{Abdul Nasir}

University of Agriculture Faisalabad

38080 Punjab, Pakistan.

Email: anawan@uaf.edu.pk

Received 10 November 2017

Revised 01 December 2017

Accepted 12 December 2017

Available online 1 January 2018

\section{ABSTRACT}

Microbial population of a given ecological niche is collectively called as microbiome. Microbiome plays a great role in remediation of heavy metal toxicity in the rhizosphere (soil attached to the plant roots) for safe food production. Rhizospheric microbiome feeds on the contaminants, degrading them for energy and reproduction. Microbial remediation could be categorized into three grades viz. natural attenuation, biostimulation, bioaugmentation. Among these three, bioaugmentation has taken more attention due to higher substrate specificity, efficiency and sustainability. Therefore, in addition to brief introduction of natural attenuation and biostimulation, the present study will discuss the basic mechanisms of how bioaugmentation effectively detoxify rhizospheric toxic metal especially arsenic (As) and how to develop novel strain for As detoxification through bioaugmentation.

\section{KEYWORDS}

Microbiome, heavy metal, rhizosphere, biostimulation, bioaugmentation.

\section{NATURAL ATTENUATION}

The process takes place naturally with indigenous soil microorganisms since the very beginning of the world. In here, natural indigenous microorganisms detoxify rhizospheric toxic substances in-vivo.

\section{BIO STIMULATION}

The microbial detoxification of rhizospheric contaminants depends on the availability of nutrients (nitrogen, carbon, phosphorus, potassium and other major nutrients), favorable environmental conditions ( $\mathrm{pH}$, organic matter, electrical conductivity, aeration, temperature), and cropping systems. Such as continuous sugar cane cropping acidifies soil, scarcity of nutrients and unfavorable conditions restrict microbial growth lead to adverse effects to microbiome mediated detoxification of rhizospheric toxic substances. In addition, the nature of contaminant itself and its bioavailability also affect the survival of rhizospheric microbiome. Therefore, providing favorable conditions to the rhizospheric microbes are the major principles of the approach.

\section{BIOAUGMENTATION}

Bioaugmentation involves the exogenous introduction of nonpathogenic microorganisms to detoxify the rhizosphere due to inefficiency of indigenous microbes in-vivo. The augmented strains must have superior performance to degrade the target contaminants than the natural, along with rapid growth, and tolerant in wide range of highly toxic environments. Bioaugmentation is commonly done by genetically engineered microbes that could survive extremely toxic environment with better performance than the natural.

\section{MICROBIAL REMEDIATION OF ARSENIC (As)}

The potential As reducing microbes efficiently reduce very toxic easily soluble forms into less toxic insoluble forms either by efflux of As ions outside the cell, or accumulating and forming complex of the As inside the cell and later reduction of As ions to a non-toxic state. Microbes could either do immobilization or mobilization during bioremediation of As.

\subsection{As immobilization by microbes}

Microbial immobilization of As involves alteration of physical or chemical properties of As to reduce its mobility. Microbial metabolites especially assist As immobilization. Microorganisms could utilize their metabolites for solidification and stabilization of As leading to immobilization. Due to precipitation of hydroxides released by microorganism, As is immobilized [1]. Microorganisms should have potential to produce appropriate amounts of stabilizer/metabolites to immobilize As.

\subsection{As mobilization by microbes}

Microbial siderophores, metabolites, methylation, and redox transformations can mobilize As by autotrophic and heterotrophic leaching, chelation [2]. Potential microbes could acidify the rhizosphere by proton efflux, resulting release of free As consequences heterotrophic leaching. Potential microorganisms could mobilize As by reduction and oxidation processes through redox transformations. By anaerobic 
respiration microorganisms could utilize As either as electron acceptor, or donor to fix $\mathrm{CO}_{2}$ into cell carbon. Microorganisms reduce arsenate to arsenite by using arsenate as an electron acceptor. Alternately, As oxidizer microorganisms could oxidizes arsenite to arsenate by utilizing the carbon from $\mathrm{CO}_{2}$ and electron from arsenite for energy $[3,4]$.

\subsection{Bioaugmentation approach to develop novel strain for rhizospheric remediation}

Microbial species with high chitin, chitosan and glucans content in cell wall act as an effective biosorbent. Hence, the microbial accumulation of intracellular As is more preferred than other mechanism of As bioremediation. However, natural strains are not so efficient to serve the purpose. Therefore, to increase efficiency, the natural strains must be engineered. For example- a strain of Corynebacterium glutamicum ( $C$. glutamicum) is engineered by the removal of the ArsC (arsenate reductase) and Acr3 (efflux) systems consequences an increased accumulation of intracellular As (28-30 folds). In addition, by deleting As(III) efflux system, As(III) biocontainers were also engineered. In $C$. glutamicum, increase uptake of arsenite was also observed by overexpression of GlpF protein too. Therefore, the genetically engineered (GF) C. glutamicum accumulated the large amount of As in two-step (second steo) detoxification system. Firstly, As oxidizer microbes (mostly Ochrobacterium tritici) oxidized $\mathrm{As}(\mathrm{III})$ to $\mathrm{As}(\mathrm{V})$ following accumulation of $\mathrm{As}(\mathrm{V})$ and remaining As(III) could be detoxified by engineered $C$. glutamicum [5]. In addition to the above information, a large number of research findings regarding use of GE microorganism for As remediation are available in recent reviews. Therefore, efficient, non pathogenic As detoxifying microbes should be isolated from the nature and proper genetic modification should be done to increase its efficiency and to serve our purpose. Some genes in As detoxifying pathway could be overexpressed to increase detoxification efficiency and feed inhibition could be optimized by silencing some of the feedback inhibition genes (Figure 1).

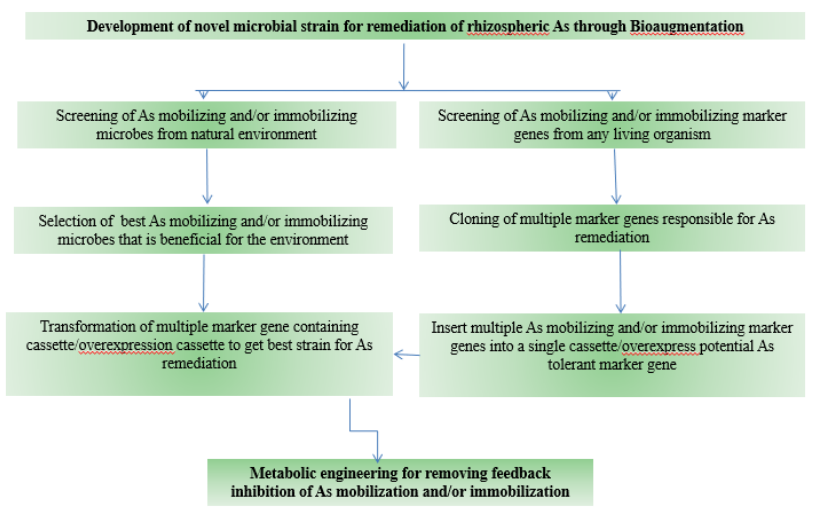

Figure 1: Proposed model for development of novel strain for rhizospheric As remediation

\section{CONCLUDING REMARKS}

Natural attenuation, biostimulation and bioaugmentation could have significant influence in rhizospheric As detoxification. However, due to intensive agriculture, mono and/or continuous cropping, and climate change the quantity and diversity of rhizospheric microbiome is decreasing. Therefore, it is a big challenge for the researcher to select proper strain from a large group of species that could be used for rhizospheric As remediation. To achieve the goal, researchers have to follow an integrative approach. Hence, the proposed model (Figure 1) could be followed to develop a novel strain that could efficiently mobilize and /or immobilize rhizospheric As for safe food crop production. However, this needs application of scientific knowledge and expertise in food microbiology, molecular biology, and nutrition of food crops in an integrative manner.

\section{REFERENCES}

[1] Zhou, J., Shu, W., Gao, Y., Cao, Z., Zhang, J., Hou, H., Zhao, J., Chen, X., Pan, Y., Qian, G. 2017. Enhanced arsenite immobilization via ternary layered double hydroxides and application to paddy soil remediation. RSC Adv., 7, 20320-20326.

[2] Huang, J.H. 2014. Impact of Microorganisms on Arsenic Biogeochemistry: A Review. Water Air Soil Pollution, 225, 1848.

[3] Stolz, J.F., Basu, P., Joanne, M.S., Oremland, R.S. 2006. Arsenic and selenium in microbial metabolism. Annual Review of Microbiology, 60, 107-30.

[4] Zhang, J., Zhou, W., Liu, B., He, J., Shen, Q., Zhao, F.J. 2015. Anaerobic Arsenite Oxidation by an Autotrophic Arsenite-Oxidizing Bacterium from an Arsenic-Contaminated Paddy Soil. Environmental Science and Technology, 49, 5956-5964.

[5] Villadangos, A.F., Ordóñez, E., Pedre, B., Messens, J., Gil, J.A., Mateos, L.M. 2014. Engineered coryneform bacteria as a bio-tool for arsenic remediation. Applied Microbiology and Biotechnology, 98, 10143-10152. 